Research Paper

\title{
LMOI super-enhancer polymorphism rs2168101 G>T correlates with decreased neuroblastoma risk in Chinese children
}

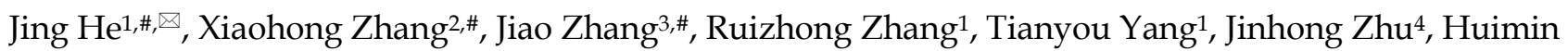
$\mathrm{Xia}^{1}, \mathrm{Yan}_{\mathrm{Z}} \mathrm{ou}^{1, \otimes}$

1. Department of Pediatric Surgery, Guangzhou Institute of Pediatrics, Guangzhou Women and Children's Medical Center, Guangzhou Medical University, Guangzhou 510623, Guangdong, China

2. Department of Hematology/Oncology, Guangzhou Women and Children's Medical Center, Guangzhou Medical University, Guangzhou 510623, Guangdong, China

3. Department of Pediatric Surgery, the First Affiliated Hospital of Zhengzhou University, Zhengzhou 450052, Henan, China

4. Molecular Epidemiology Laboratory and Department of Laboratory Medicine, Harbin Medical University Cancer Hospital, Harbin 150040, Heilongjiang, China

\# These authors contributed equally to this work.

$\triangle$ Corresponding authors: Jing He, Department of Pediatric Surgery, Guangzhou Institute of Pediatrics, Guangzhou Women and Children's Medical Center, Guangzhou Medical University, 9 Jinsui Road, Guangzhou 510623, Guangdong, China, Tel./Fax: (+86-020) 38076560, Email: hejing198374@gmail.com or hejing@gwcmc.org; or Yan Zou, Department of Pediatric Surgery, Guangzhou Women and Children's Medical Center, Guangzhou Medical University, 9 Jinsui Road, Guangzhou 510623, Guangdong, China, Tel./Fax: (+86-020) 38076146, Email: monknut@126.com.

() Ivyspring International Publisher. This is an open access article distributed under the terms of the Creative Commons Attribution (CC BY-NC) license (https://creativecommons.org/licenses/by-nc/4.0/). See http://ivyspring.com/terms for full terms and conditions.

Received: 2017.12.12; Accepted: 2018.02.06; Published: 2018.04.12

\begin{abstract}
Neuroblastoma is one of the most frequently occurring childhood cancers. The rs2168101 G>T polymorphism observed in the $L M O I$ gene is located at a conserved GATA transcription factor binding motif. This polymorphism was reported to be significantly associated with neuroblastoma susceptibility. However, whether this and other functional polymorphisms can affect neuroblastoma risk of Chinese children remains unknown. We conducted a two-center hospital-based case-control study with a total of 374 cases and 812 controls to assess the role of five LMOI gene polymorphisms in the neuroblastoma risk. We confirmed that $\mathrm{rs} 2168101 \mathrm{G}>\mathrm{T}$ was significantly associated with decreased neuroblastoma risk for both northern and southern Chinese children and the combined subjects [GT vs. GG: adjusted odds ratio $(O R)=0.57,95 \%$ confidence interval $(C l)=0.44-0.74, P<0.0001 ; T T$ vs. $G G$ : adjusted $O R=0.29,95 \%$ $\mathrm{Cl}=0.15-0.56, P=0.0002$; $\mathrm{GT} / \mathrm{TT}$ vs. GG: adjusted $\mathrm{OR}=0.53,95 \% \mathrm{Cl}=0.41-0.68, P<0.0001$; and TT vs. GT/GG: adjusted $\mathrm{OR}=0.36,95 \% \mathrm{Cl}=0.19-0.69, P=0.002$ ] after adjustment for age and gender. This association was further confirmed by performing a stratifying analysis and a false-positive report probability analysis. Similar results were observed for the rs3750952 G>C polymorphism. In summary, the current study confirmed that the potentially functional LMOI rs2168101 G>T and rs3750952 G>C polymorphisms were associated with neuroblastoma susceptibility. This research requires further validation with larger sample sizes and inclusion of different ethnicities.
\end{abstract}

Key words: LMO1; polymorphism; neuroblastoma; risk; genetic susceptibility

\section{Introduction}

Neuroblastoma is a commonly occurring pediatric tumor derived from the embryonic neural crest [1]. Neuroblastoma is the fourth most frequently occurring tumor among Chinese children with an incidence of 7.7 cases per million [2]. While low- and intermediate-risk patients may have a good prognosis, high-risk neuroblastoma is still difficult to cure. Approximately $40 \%$ of patients are high-risk and have poor outcomes $[3,4]$. Potential risk factors for children developing neuroblastoma include parental exposure to wood dust, radiation sources, solders, and hydrocarbons, though the reasons are 
incompletely understood [5, 6]. Genetic factors (e.g., mutations and polymorphisms) may also play an important role in the occurrence of neuroblastoma [7-11]. For instance, FAS/FASL gene polymorphisms has been shown to confer neuroblastoma risk in Chinese children [12]. Low-frequency variants may also contribute to tumorigenesis including neuroblastoma $[13,14]$.

The LIM domain only 1 (LMO1) gene located at the 11 p15 region encodes an intertwining LIM-only transcriptional regulator [15]. The LMO1 protein is implicated in the initiation and development of several cancers [16]. A genome-wide association study (GWAS) comprising 2,251 neuroblastoma cases and 6,097 controls of European ancestry identified four single nucleotide polymorphisms (SNPs) in the LMO1 gene that were associated with neuroblastoma susceptibility [15]. None of the four polymorphisms (rs110419 A>G, rs4758051 G>A, rs10840002 A>G and rs204938 $A>G$ ) is a potentially functional polymorphism. In 2015, Oldridge et al. [17] identified a causal DNA variant (rs2168101 G>T) in the LMO1 gene that was significantly associated with decreased neuroblastoma susceptibility (combined $P=7.47 \mathrm{x}$ $10^{-29}$ ). To date, this association has not been verified among other ethnicities; therefore, we conducted the current two-center case-control study among a total of 374 neuroblastoma cases and 812 controls.

\section{Materials and methods}

\section{Study subjects}

This study was comprised of two independent retrospective hospital-based case-control studies (Supplemental Table 1). The southern Chinese population study involved 256 neuroblastoma patients and 531 controls that were recruited from the Guangzhou Women and Children's Medical Center, as we described previously [18-20]. The northern Chinese population study involved 118 neuroblastoma patients and 281 controls that were recruited from the First Affiliated Hospital of Zhengzhou University between August 2011 and April 2017 [21, 22]. Briefly, all of the included cases were histopathologically diagnosed as new neuroblastoma patients. All of the included subjects provided written informed consent by their guardian. This study was authorized by the Institutional Review Board of each hospital.

\section{Selection of polymorphisms and genotyping}

Five polymorphisms (rs2168101 G>T, rs1042359 A > G, rs11041838 G>C, rs2071458 C>A, and rs3750952 $\mathrm{G}>\mathrm{C}$ ) in the LMO1 gene were selected (Supplemental Table 2). Of the five polymorphisms, the rs 2168101 $\mathrm{G}>\mathrm{T}, \quad \mathrm{rs} 1042359 \quad \mathrm{~A}>\mathrm{G}, \quad$ and $\mathrm{rs3750952} \quad \mathrm{G}>\mathrm{C}$ polymorphisms were identified in a previous study [17]. We also chose rs11041838 G>C and rs2071458 C>A, which were potentially functional (Transcription factor binding site, TFBS) polymorphisms according to the online software SNPinfo (https://snpinfo.niehs.nih.gov/). Genomic DNA was primarily extracted from peripheral blood lymphocytes. All five selected polymorphisms were genotyped by the Taqman methodology, as described previously $[23,24]$. At least $10 \%$ of the samples were randomly selected for further confirmation, and the results were $100 \%$ concordant [25-27].

\section{Statistical analysis}

The Chi-square test was adopted to compare the frequency distribution of demographic variables and genotypes. The goodness-of- $\chi^{2}$ test was used to evaluate the Hardy-Weinberg equilibrium (HWE) for control subjects. Multivariate logistic regression was used to calculate the odds ratio (OR) and the 95\% confidence interval (CI). We also calculated the false-positive report probability (FPRP) values for significant findings from the combined subjects, as we described previously $[28,29]$. $P$ values less than 0.05 were considered to be statistically significant findings.

\section{Results}

\section{Characteristics of subjects}

As shown in Supplemental Table 1, no significant difference was observed between cases and controls in age $(P=0.239)$ or gender $(P=0.333)$ for southern Chinese subjects. Similar findings were observed among the northern Chinese children ( $P=0.189$ for age, and $P=0.196$ for gender).

\section{LMOI gene polymorphisms and neuroblastoma susceptibility}

As shown in Table 1, the genotype frequencies of the five selected polymorphisms did not deviate from the HWE in the combined controls $(P=0.448$ for rs2168101 $\mathrm{G}>\mathrm{T}, P=0.786$ for $\mathrm{rs} 1042359 \mathrm{~A}>\mathrm{G}, P=0.056$ for rs11041838 $\mathrm{G}>\mathrm{C}, P=0.427$ for rs2071458 $\mathrm{C}>\mathrm{A}$, and $P=0.886$ for rs3750952 $\mathrm{G}>\mathrm{C}$ ). Regarding the selected polymorphisms, after adjustment for age and gender, we observed that the rs2168101 G>T polymorphism was significantly associated with decreased neuroblastoma risk for southern Chinese subjects (GT vs. GG: adjusted $\mathrm{OR}=0.62,95 \% \mathrm{CI}=0.45-0.86, P=0.004$; TT vs. GG: adjusted $\mathrm{OR}=0.29,95 \% \mathrm{CI}=0.13-0.67$, $P=0.004 ;$ GT/TT vs. GG: adjusted $\mathrm{OR}=0.57,95 \%$ $\mathrm{CI}=0.42-0.78, P=0.0004$; and TT vs. GT/GG: adjusted $\mathrm{OR}=0.35,95 \% \mathrm{CI}=0.15-0.80, P=0.012)$, and for northern Chinese subjects (GT vs. GG: adjusted OR $=0.45,95 \%$ $\mathrm{CI}=0.28-0.73, P=0.001$; TT vs. $\mathrm{GG}$ : adjusted $\mathrm{OR}=0.26$, 95\% CI=0.09-0.78, $P=0.016$; and GT/TT vs. GG: 
adjusted $\mathrm{OR}=0.42,95 \% \mathrm{CI}=0.27-0.66, P=0.0002)$. When we combined all subjects, the protective effect was more noteworthy (GT vs. GG: adjusted OR $=0.57,95 \%$ $\mathrm{CI}=0.44-0.74, P<0.0001$; TT vs. GG: adjusted $\mathrm{OR}=0.29$, 95\% CI=0.15-0.56, $P=0.0002$; GT/TT vs. GG: adjusted $\mathrm{OR}=0.53,95 \% \mathrm{CI}=0.41-0.68, \quad P<0.0001$; and $\mathrm{TT}$ vs. GT/GG: adjusted $\mathrm{OR}=0.36, \quad 95 \% \quad \mathrm{CI}=0.19-0.69$, $P=0.002)$, after adjustment for age and gender. Similar significant associations were found for the rs3750952 G>C polymorphism for the southern, northern and combined subjects. When the protective genotypes were combined, we observed that the $2-5$ protective genotype carriers had a significantly decreased neuroblastoma risk for southern (adjusted $\mathrm{OR}=0.68$, 95\% $\quad \mathrm{CI}=0.50-0.92, \quad P=0.012$ ), northern (adjusted $\mathrm{OR}=0.53,95 \% \mathrm{CI}=0.34-0.83, P=0.005)$ and combined subjects (adjusted OR=0.62, 95\% CI $=0.49-0.80$, $P=0.0002)$, after adjustment for age and gender.

\section{Stratified analysis}

The associations between rs2168101 G>T, rs3750952 G>C, and the combined protective genotypes with neuroblastoma susceptibility were further evaluated by stratification analysis for southern Chinese subjects (Supplemental Table 3), northern Chinese subjects (Supplemental Table 4), and combined subjects (Table 2). For the rs2168101 G>T polymorphism, the protective effect was prominent among children older than 18 months, males, females, tumors that originated from the adrenal gland and the mediastinum, and both the INSS stage I+II+4s, and stage III+IV subjects. A similar significant association was observed for the rs3750952 G>C polymorphism and the combined protective genotypes.

Table 1. Genotype frequencies of $L M O I$ gene polymorphisms and neuroblastoma susceptibility

\begin{tabular}{|c|c|c|c|c|c|c|c|c|c|c|c|c|}
\hline \multirow[t]{2}{*}{ Genotype } & \multicolumn{4}{|c|}{ Guangdong province } & \multicolumn{4}{|c|}{ Henan province } & \multicolumn{4}{|l|}{ Combined } \\
\hline & $\begin{array}{l}\text { Cases } \\
(\mathrm{N}=255)\end{array}$ & $\begin{array}{l}\text { Controls } \\
(\mathrm{N}=531)\end{array}$ & $\begin{array}{l}\text { Adjusted OR } \\
(95 \% \mathrm{CI})^{\mathrm{a}}\end{array}$ & $P a$ & $\begin{array}{l}\text { Cases } \\
(\mathrm{N}=118)\end{array}$ & $\begin{array}{l}\text { Controls } \\
(\mathrm{N}=281)\end{array}$ & $\begin{array}{l}\text { Adjusted OR } \\
(95 \% \mathrm{CI})^{\text {a }}\end{array}$ & $P a$ & $\begin{array}{l}\text { Cases } \\
(\mathrm{N}=373)\end{array}$ & $\begin{array}{l}\text { Controls } \\
(\mathrm{N}=812)\end{array}$ & $\begin{array}{l}\text { Adjusted OR } \\
(95 \% \mathrm{CI})^{\text {a }}\end{array}$ & $P a$ \\
\hline \multicolumn{5}{|c|}{ rs2168101 G>T (HWE=0.670) } & \multicolumn{4}{|l|}{$\mathrm{HWE}=0.462$} & \multicolumn{4}{|l|}{$\mathrm{HWE}=0.448$} \\
\hline GG & $166(65.10)$ & $275(51.79)$ & 1.00 & & $79(66.95)$ & $132(46.98)$ & 1.00 & & $245(65.68)$ & 407 (50.12) & 1.00 & \\
\hline GT & $82(32.16)$ & $217(40.87)$ & $0.62(0.45-0.86)$ & 0.004 & $35(29.66)$ & $125(44.48)$ & $0.45(0.28-0.73)$ & 0.001 & $117(31.37)$ & $342(42.12)$ & $0.57(0.44-0.74)$ & $<0.0001$ \\
\hline TT & $7(2.75)$ & $39(7.34)$ & $0.29(0.13-0.67)$ & 0.004 & $4(3.39)$ & $24(8.54)$ & $0.26(0.09-0.78)$ & 0.016 & $11(2.95)$ & $63(7.76)$ & $0.29(0.15-0.56)$ & 0.0002 \\
\hline Additive & & & $0.59(0.45-0.77)$ & 0.0001 & & & $0.47(0.32-0.70)$ & 0.0002 & & & $0.56(0.45-0.69)$ & $<0.0001$ \\
\hline Dominant & $89(34.90)$ & $256(48.21)$ & $0.57(0.42-0.78)$ & 0.0004 & $39(33.05)$ & $149(53.02)$ & $0.42(0.27-0.66)$ & 0.0002 & $128(34.32)$ & $405(49.88)$ & $0.53(0.41-0.68)$ & $<0.0001$ \\
\hline Recessive & $248(97.25)$ & $492(92.66)$ & $0.35(0.15-0.80)$ & 0.012 & $114(96.61)$ & $257(91.46)$ & $0.36(0.12-1.05)$ & 0.062 & $362(97.05)$ & $749(92.24)$ & $0.36(0.19-0.69)$ & 0.002 \\
\hline \multicolumn{5}{|c|}{ rs1042359 A > G $(H W E=0.065)$} & \multicolumn{4}{|c|}{$\mathrm{HWE}=0.199$} & \multicolumn{4}{|c|}{$\mathrm{HWE}=0.786$} \\
\hline AA & $233(91.37)$ & $485(91.34)$ & 1.00 & & $97(82.20)$ & $224(79.72)$ & 1.00 & & $330(88.47)$ & 709 (87.32) & 1.00 & \\
\hline AG & $21(8.24)$ & $43(8.10)$ & $1.02(0.60-1.76)$ & 0.947 & $20(16.95)$ & $56(19.93)$ & $0.82(0.47-1.44)$ & 0.491 & $41(10.99)$ & 99 (12.19) & $0.89(0.60-1.31)$ & 0.547 \\
\hline GG & $1(0.39)$ & $3(0.56)$ & $0.69(0.07-6.70)$ & 0.750 & $1(0.85)$ & $1(0.36)$ & $2.26(0.14-36.68)$ & 0.567 & $2(0.54)$ & $4(0.49)$ & $1.08(0.20-5.94)$ & 0.928 \\
\hline Additive & & & $0.98(0.60-1.60)$ & 0.933 & & & $0.88(0.52-1.50)$ & 0.643 & & & $0.91(0.64-1.30)$ & 0.606 \\
\hline Dominant & $22(8.63)$ & $46(8.66)$ & $1.00(0.59-1.70)$ & 0.992 & $21(17.80)$ & $57(20.28)$ & $0.85(0.49-1.47)$ & 0.554 & $43(11.53)$ & $103(12.68)$ & $0.90(0.61-1.31)$ & 0.568 \\
\hline Recessive & $254(99.61)$ & $528(99.44)$ & $0.69(0.07-6.68)$ & 0.749 & $117(99.15)$ & $280(99.64)$ & $2.34(0.14-37.99)$ & 0.550 & $371(99.46)$ & $808(99.51)$ & $1.10(0.20-6.02)$ & 0.915 \\
\hline \multicolumn{5}{|c|}{ rs11041838 G>C $(\mathrm{HWE}=0.448)$} & \multicolumn{4}{|l|}{$\mathrm{HWE}=0.031$} & \multicolumn{4}{|l|}{$\mathrm{HWE}=0.056$} \\
\hline GG & $175(68.63)$ & $386(72.69)$ & 1.00 & & $85(72.03)$ & $196(69.75)$ & 1.00 & & $260(69.71)$ & $582(71.67)$ & 1.00 & \\
\hline GC & $74(29.02)$ & $136(25.61)$ & $1.20(0.86-1.68)$ & 0.290 & $31(26.27)$ & $83(29.54)$ & $0.88(0.54-1.44)$ & 0.614 & $105(28.15)$ & $219(26.97)$ & $1.08(0.82-1.42)$ & 0.608 \\
\hline $\mathrm{CC}$ & $6(2.35)$ & $9(1.69)$ & $1.46(0.51-4.18)$ & 0.478 & $2(1.69)$ & $2(0.71)$ & $2.19(0.30-16.01)$ & 0.440 & $8(2.14)$ & $11(1.35)$ & $1.62(0.65-4.08)$ & 0.304 \\
\hline Additive & & & $1.20(0.90-1.61)$ & 0.221 & & & $0.96(0.61-1.51)$ & 0.867 & & & $1.12(0.88-1.43)$ & 0.370 \\
\hline Dominant & $80(31.37)$ & $145(27.31)$ & $1.22(0.88-1.68)$ & 0.243 & $33(27.97)$ & $85(30.25)$ & $0.92(0.57-1.48)$ & 0.719 & $113(30.29)$ & $230(28.33)$ & $1.10(0.84-1.44)$ & 0.483 \\
\hline Recessive & $249(97.65)$ & $522(98.31)$ & $1.39(0.49-3.95)$ & 0.536 & $116(98.31)$ & 279 (99.29) & $2.26(0.31-16.46)$ & 0.421 & $365(97.86)$ & $801(98.65)$ & $1.59(0.63-3.99)$ & 0.323 \\
\hline \multicolumn{5}{|c|}{ rs2071458 C>A (HWE=0.911) } & \multicolumn{4}{|l|}{$\mathrm{HWE}=0.320$} & \multicolumn{4}{|l|}{$\mathrm{HWE}=0.427$} \\
\hline $\mathrm{CC}$ & $160(62.75)$ & $324(61.02)$ & 1.00 & & $70(59.32)$ & $155(55.16)$ & 1.00 & & $230(61.66)$ & 479 (58.99) & 1.00 & \\
\hline CA & $81(31.76)$ & $181(34.09)$ & $0.91(0.66-1.25)$ & 0.551 & $42(35.59)$ & $103(36.65)$ & $0.92(0.58-1.46)$ & 0.736 & $123(32.98)$ & $284(34.98)$ & $0.90(0.69-1.17)$ & 0.439 \\
\hline AA & $14(5.49)$ & $26(4.90)$ & $1.13(0.57-2.22)$ & 0.733 & $6(5.08)$ & $23(8.19)$ & $0.58(0.23-1.50)$ & 0.264 & $20(5.36)$ & $49(6.03)$ & $0.85(0.49-1.47)$ & 0.560 \\
\hline Additive & & & $0.98(0.76-1.26)$ & 0.843 & & & $0.84(0.59-1.19)$ & 0.331 & & & $0.91(0.74-1.12)$ & 0.375 \\
\hline Dominant & $95(37.25)$ & $207(38.98)$ & $0.93(0.69-1.27)$ & 0.661 & $48(40.68)$ & $126(44.84)$ & $0.86(0.56-1.34)$ & 0.504 & $143(38.34)$ & $333(41.01)$ & $0.89(0.70-1.15)$ & 0.381 \\
\hline Recessive & $241(94.51)$ & $505(95.10)$ & $1.17(0.60-2.28)$ & 0.656 & $112(94.92)$ & $258(91.81)$ & $0.60(0.24-1.52)$ & 0.283 & $353(94.64)$ & $763(93.97)$ & $0.88(0.52-1.51)$ & 0.649 \\
\hline \multicolumn{5}{|c|}{ rs3750952 G>C $(\mathrm{HWE}=0.994)$} & \multicolumn{4}{|l|}{$\mathrm{HWE}=0.763$} & \multicolumn{4}{|l|}{$\mathrm{HWE}=0.886$} \\
\hline GG & $147(57.65)$ & $253(47.65)$ & 1.00 & & $65(55.08)$ & $120(42.70)$ & 1.00 & & $212(56.84)$ & $373(45.94)$ & 1.00 & \\
\hline GC & $100(39.22)$ & $227(42.75)$ & $0.76(0.56-1.03)$ & 0.081 & $47(39.83)$ & $129(45.91)$ & $0.66(0.42-1.03)$ & 0.069 & $147(39.41)$ & $356(43.84)$ & $0.73(0.56-0.94)$ & 0.014 \\
\hline $\mathrm{CC}$ & $8(3.14)$ & $51(9.60)$ & $0.27(0.12-0.58)$ & 0.0008 & $6(5.08)$ & $32(11.39)$ & $0.32(0.13-0.81)$ & 0.017 & $14(3.75)$ & $83(10.22)$ & $0.30(0.16-0.53)$ & $<0.0001$ \\
\hline Additive & & & $0.65(0.50-0.83)$ & 0.0006 & & & $0.61(0.43-0.87)$ & 0.006 & & & $0.64(0.52-0.78)$ & $<0.0001$ \\
\hline Dominant & $108(42.35)$ & $278(52.35)$ & $0.67(0.49-0.90)$ & 0.008 & $53(44.92)$ & $161(57.30)$ & $0.59(0.38-0.91)$ & 0.018 & $161(43.16)$ & $439(54.06)$ & $0.65(0.50-0.83)$ & 0.0005 \\
\hline Recessive & $247(96.86)$ & $480(90.40)$ & $0.30(0.14-0.64)$ & 0.002 & $112(94.92)$ & $249(88.61)$ & $0.39(0.16-0.97)$ & 0.043 & $359(96.25)$ & $729(89.78)$ & $0.34(0.19-0.61)$ & 0.0003 \\
\hline \multicolumn{13}{|c|}{ Combined effect of protective genotypes ${ }^{c}$} \\
\hline $0-1$ & $129(50.59)$ & $218(41.05)$ & 1.00 & & $55(46.61)$ & 89 (31.67) & 1.00 & & $184(49.33)$ & 307 (37.81) & 1.00 & \\
\hline $2-5$ & $126(49.41)$ & 313 (58.95) & $0.68(0.50-0.92)$ & 0.012 & 63 (53.39) & $192(68.33)$ & $0.53(0.34-0.83)$ & 0.005 & $189(50.67)$ & 505 (62.19) & $0.62(0.49-0.80)$ & 0.0002 \\
\hline
\end{tabular}




\section{FPRP analysis}

The FPRP results for statistically significant findings from the combined subjects are shown in Table 3. When we adopted a FPRP threshold of 0.2, as suggested by Wacholder et al. [30], we observed that all of the significant findings for the rs2168101 still remained noteworthy at the prior probability of 0.1 , except for rs2168101 TT vs. GG/GT. Similar results were observed for rs3750952 G>C, with exceptions of females, tumor origin from the adrenal gland and INSS stage I+II+4s. Regarding the combined protective genotypes, all of the significant findings were still noteworthy, except for males and tumor originated from the adrenal gland.

Table 2. Stratification analysis for the association between LMOI gene polymorphisms and neuroblastoma susceptibility (combined subjects)

\begin{tabular}{|c|c|c|c|c|c|c|c|c|c|c|c|c|}
\hline \multirow{3}{*}{ Variables } & \multicolumn{4}{|c|}{ rs2168101 G>T } & \multicolumn{4}{|c|}{ rs3750952 G>C } & \multicolumn{4}{|c|}{ Protective genotypes ${ }^{a}$} \\
\hline & GG & $\mathrm{GT} / \mathrm{TT}$ & Adjusted OR ${ }^{b}$ & $P_{\mathrm{b}}$ & GG & $\mathrm{GC} / \mathrm{CC}$ & Adjusted OR ${ }^{\mathrm{b}}$ & $P$ b & $0-1$ & $2-5$ & Adjusted OR ${ }^{b}$ & $P \mathrm{~b}$ \\
\hline & \multicolumn{2}{|c|}{ (Cases/Controls) } & \multicolumn{2}{|l|}{$(95 \% \mathrm{CI})$} & \multicolumn{2}{|c|}{ (Cases/Controls) } & \multicolumn{2}{|l|}{$(95 \% \mathrm{CI})$} & \multicolumn{2}{|c|}{ (Cases/Controls) } & \multicolumn{2}{|l|}{$(95 \%$ CI $)$} \\
\hline \multicolumn{13}{|l|}{ Age, month } \\
\hline$\leq 18$ & $77 / 158$ & $47 / 147$ & $0.65(0.43-1.00)$ & 0.052 & $64 / 146$ & $60 / 159$ & $0.86(0.57-1.31)$ & 0.477 & $58 / 118$ & $66 / 187$ & $0.72(0.47-1.09)$ & 0.123 \\
\hline$>18$ & $168 / 249$ & $81 / 258$ & $0.46(0.34-0.64)$ & $<0.0001$ & $148 / 227$ & $101 / 280$ & $0.55(0.40-0.75)$ & 0.0001 & $126 / 189$ & $123 / 318$ & $0.58(0.43-0.79)$ & 0.0005 \\
\hline \multicolumn{13}{|l|}{ Gender } \\
\hline Females & $107 / 166$ & $50 / 176$ & $0.44(0.30-0.66)$ & $<0.0001$ & $88 / 152$ & $69 / 190$ & $0.63(0.43-0.92)$ & 0.018 & $81 / 127$ & $76 / 215$ & $0.56(0.38-0.81)$ & 0.003 \\
\hline Males & $138 / 241$ & $78 / 229$ & $0.60(0.43-0.83)$ & 0.002 & $124 / 221$ & $92 / 249$ & $0.66(0.48-0.92)$ & 0.013 & $103 / 180$ & $113 / 290$ & $0.68(0.49-0.94)$ & 0.021 \\
\hline \multicolumn{13}{|l|}{ Sites of origin } \\
\hline Adrenal gland & $95 / 407$ & $39 / 405$ & $0.41(0.28-0.61)$ & $<0.0001$ & $77 / 373$ & $57 / 439$ & $0.63(0.43-0.91)$ & 0.013 & $63 / 307$ & $71 / 505$ & $0.68(0.47-0.99)$ & 0.044 \\
\hline Retroperitoneal & $53 / 407$ & $34 / 405$ & $0.65(0.41-1.02)$ & 0.063 & $43 / 373$ & $44 / 439$ & $0.88(0.56-1.37)$ & 0.566 & $38 / 307$ & $49 / 505$ & $0.79(0.51-1.24)$ & 0.300 \\
\hline Mediastinum & $74 / 407$ & $35 / 405$ & $0.48(0.31-0.73)$ & 0.0006 & $71 / 373$ & $38 / 439$ & $0.46(0.30-0.69)$ & 0.0002 & $67 / 307$ & $42 / 505$ & $0.38(0.25-0.58)$ & $<0.0001$ \\
\hline Others & $21 / 407$ & $14 / 405$ & $0.67(0.34-1.34)$ & 0.259 & $18 / 373$ & $17 / 439$ & $0.81(0.41-1.59)$ & 0.535 & $14 / 307$ & $21 / 505$ & $0.91(0.46-1.82)$ & 0.796 \\
\hline \multicolumn{13}{|l|}{ Clinical stages } \\
\hline $\mathrm{I}+\mathrm{II}+4 \mathrm{~s}$ & $103 / 407$ & $61 / 405$ & $0.60(0.42-0.84)$ & 0.003 & $90 / 373$ & $74 / 439$ & $0.70(0.50-0.98)$ & 0.036 & $81 / 307$ & $83 / 505$ & $0.62(0.44-0.87)$ & 0.006 \\
\hline $\mathrm{III}+\mathrm{IV}$ & $127 / 407$ & $62 / 405$ & $0.48(0.35-0.68)$ & $<0.0001$ & $110 / 373$ & $79 / 439$ & $0.60(0.44-0.83)$ & 0.002 & $91 / 307$ & $98 / 505$ & $0.65(0.47-0.89)$ & 0.008 \\
\hline
\end{tabular}

OR, odds ratio; $\mathrm{CI}$, confidence interval.

a Protective genotypes that decreased neuroblastoma risk were rs2168101 GT/TT, rs1042359 AG/GG, rs11041838 GG, rs2071458 CA/AA, and rs3750952 GC/CC.

${ }^{\mathrm{b}}$ Adjusted for age and gender, omitting the corresponding stratification factor.

Table 3. False-positive report probability results for outstanding findings in combined subjects

\begin{tabular}{|c|c|c|c|c|c|c|c|c|}
\hline \multirow[t]{2}{*}{ Genotype } & \multirow{2}{*}{$\begin{array}{l}\text { Crude OR } \\
(95 \% \text { CI })\end{array}$} & \multirow[t]{2}{*}{$P$ a } & \multirow[t]{2}{*}{ Statistical power ${ }^{b}$} & \multicolumn{5}{|c|}{ Prior probability } \\
\hline & & & & 0.25 & 0.1 & 0.01 & 0.001 & 0.0001 \\
\hline \multicolumn{9}{|l|}{ rs2168101 G>T } \\
\hline GT vs. GG & $0.57(0.44-0.74)$ & $<0.0001$ & 0.122 & 0.001 & 0.002 & 0.020 & 0.170 & 0.672 \\
\hline TT vs. GG & $0.29(0.15-0.56)$ & 0.0002 & 0.009 & 0.060 & 0.160 & 0.677 & 0.955 & 0.995 \\
\hline GT/TT vs. GG & $0.53(0.41-0.68)$ & $<0.0001$ & 0.034 & 0.000 & 0.000 & 0.002 & 0.020 & 0.166 \\
\hline TT vs. GG/GT & $0.36(0.19-0.69)$ & 0.0022 & 0.050 & 0.117 & 0.284 & 0.814 & 0.978 & 0.998 \\
\hline \multicolumn{9}{|l|}{ GT/TT vs. GG } \\
\hline$>18$ & $0.47(0.34-0.64)$ & $<0.0001$ & 0.015 & 0.000 & 0.001 & 0.016 & 0.137 & 0.614 \\
\hline Females & $0.44(0.30-0.66)$ & $<0.0001$ & 0.023 & 0.007 & 0.021 & 0.188 & 0.700 & 0.959 \\
\hline Males & $0.60(0.43-0.83)$ & 00.0021 & 0.247 & 0.025 & 0.071 & 0.457 & 0.895 & 0.988 \\
\hline Adrenal gland & $0.41(0.28-0.61)$ & $<0.0001$ & 0.011 & 0.003 & 0.010 & 0.100 & 0.529 & 0.918 \\
\hline Mediastinum & $0.48(0.31-0.73)$ & 0.0006 & 0.065 & 0.027 & 0.078 & 0.482 & 0.904 & 0.989 \\
\hline $\mathrm{I}+\mathrm{II}+4 \mathrm{~s}$ & $0.60(0.42-0.84)$ & 0.0032 & 0.258 & 0.036 & 0.100 & 0.551 & 0.925 & 0.992 \\
\hline $\mathrm{III}+\mathrm{IV}$ & $0.49(0.35-0.69)$ & $<0.0001$ & 0.039 & 0.002 & 0.007 & 0.068 & 0.422 & 0.880 \\
\hline \multicolumn{9}{|l|}{ rs3750952 G>C } \\
\hline GC vs. GG & $0.73(0.56-0.94)$ & 0.0143 & 0.743 & 0.055 & 0.148 & 0.656 & 0.951 & 0.995 \\
\hline CC vs. GG & $0.30(0.16-0.54)$ & $<0.0001$ & 0.007 & 0.023 & 0.065 & 0.434 & 0.886 & 0.987 \\
\hline GC/CC vs. GG & $0.65(0.50-0.83)$ & 0.0005 & 0.387 & 0.004 & 0.012 & 0.116 & 0.569 & 0.930 \\
\hline CC vs. GG/GC & $0.34(0.19-0.61)$ & 0.0003 & 0.022 & 0.038 & 0.107 & 0.567 & 0.930 & 0.993 \\
\hline \multicolumn{9}{|l|}{ GC/CC vs. GG } \\
\hline$>18$ & $0.55(0.41-0.75)$ & 0.0002 & 0.118 & 0.004 & 0.013 & 0.124 & 0.589 & 0.935 \\
\hline Females & $0.63(0.43-0.92)$ & 0.0163 & 0.370 & 0.117 & 0.284 & 0.813 & 0.978 & 0.998 \\
\hline Males & $0.66(0.48-0.91)$ & 0.0117 & 0.461 & 0.071 & 0.186 & 0.715 & 0.962 & 0.996 \\
\hline Adrenal gland & $0.63(0.44-0.91)$ & 0.0138 & 0.374 & 0.100 & 0.249 & 0.785 & 0.974 & 0.997 \\
\hline Mediastinum & $0.46(0.30-0.69)$ & 0.0002 & 0.040 & 0.016 & 0.046 & 0.345 & 0.841 & 0.982 \\
\hline $\mathrm{I}+\mathrm{II}+4 \mathrm{~s}$ & $0.70(0.50-0.98)$ & 0.037 & 0.598 & 0.157 & 0.358 & 0.860 & 0.984 & 0.998 \\
\hline $\mathrm{III}+\mathrm{IV}$ & $0.61(0.44-0.84)$ & 0.0025 & 0.290 & 0.025 & 0.072 & 0.461 & 0.896 & 0.989 \\
\hline $2-5$ vs. $0-1$ & $0.62(0.49-0.80)$ & 0.0002 & 0.294 & 0.002 & 0.006 & 0.063 & 0.403 & 0.871 \\
\hline$>18$ & $0.58(0.43-0.79)$ & 0.0005 & 0.184 & 0.008 & 0.025 & 0.220 & 0.740 & 0.966 \\
\hline Females & $0.55(0.38-0.81)$ & 0.0025 & 0.168 & 0.042 & 0.117 & 0.593 & 0.936 & 0.993 \\
\hline Males & $0.68(0.49-0.94)$ & 0.0206 & 0.542 & 0.103 & 0.256 & 0.791 & 0.974 & 0.997 \\
\hline Adrenal gland & $0.69(0.47-0.99)$ & 0.0438 & 0.551 & 0.193 & 0.418 & 0.888 & 0.988 & 0.999 \\
\hline Mediastinum & $0.38(0.25-0.58)$ & $<0.0001$ & 0.005 & 0.003 & 0.008 & 0.080 & 0.466 & 0.897 \\
\hline $\mathrm{I}+\mathrm{II}+4 \mathrm{~s}$ & $0.62(0.45-0.87)$ & 0.0060 & 0.341 & 0.050 & 0.136 & 0.634 & 0.946 & 0.994 \\
\hline III+IV & $0.66(0.48-0.90)$ & 0.0092 & 0.446 & 0.057 & 0.155 & 0.668 & 0.953 & 0.995 \\
\hline
\end{tabular}

$\mathrm{OR}$, odds ratio; $\mathrm{CI}$, confidence interval.

a Chi-square test was used to calculate the genotype frequency distributions.

bStatistical power was calculated using the number of observations in the subgroup and the OR and $P$ values in this table. 


\section{Discussion}

In the current two-center hospital-based case-control study, we confirmed that the LMO1 gene rs2168101 G>T and rs3750952 G>C polymorphisms were significantly associated with decreased neuroblastoma risk for Chinese children from North and South China. To the best of our knowledge, this report describes the first replication study for the latest finding that the LMO1 super-enhancer polymorphism rs2168101 G>T was associated with a significantly decreased neuroblastoma risk.

The LMO1 gene (gene ID: 4004) is located on chromosome 11 in the p15.4 region. LMO1 encodes a cysteine-rich transcriptional regulator, which constitutes two LIM zinc-binding domains [31]. LMO1 belongs to the LMO family, which contains four superfamily numbers, LMO1 to LMO4 [31, 32]. LMO1 was reported to be mainly expressed in the nervous system and is involved in the development of the nervous system [33]. It is also reported to be highly expressed in the skin, testis, brain, adrenal and kidney tissues, as was determined through RNA-seq data performed with tissue samples derived from 95 individuals, representing 27 different tissues [34].

The LMO1 gene contains at least 5,271 polymorphisms (https://www.ncbi.nlm.nih.gov/ SNP/snp_ref.cgi?locusId=4004). In 2011, Wang et al. [15] performed a large GWAS with 2,251 neuroblastoma patients and 6,097 controls. Four LMO1 gene polymorphisms (rs110419 A>G, rs4758051 $\mathrm{G}>\mathrm{A}, \quad \mathrm{rs} 10840002 \mathrm{~A}>\mathrm{G}$ and $\mathrm{rs} 204938 \mathrm{~A}>\mathrm{G}$ ) were identified and were observed to be significantly associated with neuroblastoma susceptibility. The most significant association was with rs110419 A>G (combined $P=5.2 \times 10^{-16}$ ). These researchers also observed that LMO1 depletion may inhibit the growth of neuroblastoma cells, whereas overexpression may lead to enhanced proliferation [15]. The association between these four polymorphisms and neuroblastoma susceptibility have been validated in African-Americans [35], Italians [36], and northern [37] and southern Chinese populations [18]. The study pertaining to the African-Americans investigated 390 neuroblastoma cases and 2,500 controls. However, in this study, Latorre et al. [35] found that none of these four polymorphisms was associated with neuroblastoma susceptibility. In the study conducted by Capasso et al. [36], 370 cases and 809 controls from Italy were investigated, and it was observed that the rs110419 A>G polymorphism was associated with neuroblastoma susceptibility. However, these researchers failed to verify the association between the rs4758051 G>A polymorphism and neuroblastoma risk. In the study that investigated 244 patients and 305 controls from the northern Chinese population, Lu et al. [37] identified 11 polymorphisms within the LMO1 gene that were associated with neuroblastoma susceptibility. The most prominent polymorphism was rs204926 $\mathrm{T}>\mathrm{C} \quad(\mathrm{OR}=0.45,95 \%$ $\mathrm{CI}=0.31-0.65)$. In one of our previous publications, we included a total of 256 patients and 531 controls from South China to validate the association between these four polymorphisms (rs110419 A>G, rs4758051 G>A, rs10840002 A>G and rs204938 A>G) and neuroblastoma susceptibility, and we only verified that the rs110419 A>G polymorphism was significantly associated with neuroblastoma susceptibility [18]. However, all these four polymorphisms were not functional.

At the end of 2015, Oldridge et al. [17] investigated the causal DNA variant in the LMO1 gene and the possible mechanism by which the polymorphism may lead to neuroblastoma tumourigenesis. All possible genotypes within the LMO1 gene were determined, and highly associated polymorphisms were categorized based on chromatin accessibility and evolutionary conservation, as well as transcription factor binding site areas. These researchers observed that the LMO1 rs2168101 G>T polymorphism located at the first intron was the most highly associated variant (combined $P=7.47 \times 10^{-29}$ ). This polymorphism was located in a super-enhancer defined by extensive acetylation of histone H3 lysine 27. The LMO1 rs2168101 G allele was associated with tumor formation, a decreased event-free survival and overall survival in the European-American subjects. This association was not replicated in any other race or centers to date. We conducted the current study with neuroblastoma patients from both northern and southern Chinese populations. We verified that both the LMO1 rs2168101 G>T and rs3750952 G>C polymorphisms were significantly associated with decreased neuroblastoma susceptibility, a finding similar to that of Oldridge et al. [17]. This result indicates that the roles of these two polymorphisms are not limited to ethnicity.

Though this report describes the first replication study involving neuroblastoma patients from both North and South China, several limitations should be noted. First, although we included neuroblastoma patients from two medical centers, the sample size is still relatively small with 374 cases and 812 controls. This sample size is mainly due to the availability of samples and the low occurrence rate of neuroblastoma. Larger multinational/muticenter studies were encouraged to validate the findings. Second, only five potentially functional polymorphisms were investigated in the current study. More polymorphisms should be evaluated. Finally, due to the 
limitation of retrospective studies, several important factors, such as the parents' exposure to several environmental factors (e.g., wood dust, radiation sources, solders, and hydrocarbons), as well as the children's dietary intakes, were not available and adjusted for.

In summary, this study's results confirmed that the LMO1 gene rs2168101 G>T and rs3750952 G>C polymorphisms were associated with decreased neuroblastoma risk. These findings must be further validated through multi-center studies with large sample sizes and different ethnicities.

\section{Abbreviations}

LMO1, LIM domain only 1; GWAS, genomewide association study; SNP, single nucleotide polymorphism; HWE, Hardy-Weinberg equilibrium; OR, odds ratio; CI, confidence interval; FPRP, false-positive report probability.

\section{Supplementary Material}

Supplementary tables.

http://www.jcancer.org/v09p1592s1.pdf

\section{Acknowledgements}

This work was supported by grants from the Pearl River S\&T Nova Programme of Guangzhou (No: 201710010086), the National Natural Science Foundation of China (No: 81502046, 81602199), the State Clinical Key Specialty Construction Project (Paediatric Surgery) 2013 (No: GJLCZD1301), and Science and Technology Planning Project of Guangdong Province, China (No: 2011B032000002).

\section{Competing Interests}

The authors have declared that no competing interest exists.

\section{References}

1. Maris JM, Hogarty MD, Bagatell R, Cohn SL. Neuroblastoma. Lancet. 2007; 369: 2106-20.

2. Bao PP, Li K, Wu CX, Huang ZZ, Wang CF, Xiang YM, et al. [Recent incidences and trends of childhood malignant solid tumors in Shanghai, 2002-2010]. Zhonghua Er Ke Za Zhi. 2013; 51: 288-94.

3. Keshelava N, Zuo JJ, Chen P, Waidyaratne SN, Luna MC, Gomer CJ, et al. Loss of p53 function confers high-level multidrug resistance in neuroblastoma cell lines. Cancer Res. 2001; 61: 6185-93.

4. Bernstein ML, Leclerc JM, Bunin G, Brisson L, Robison L, Shuster J, et al. A population-based study of neuroblastoma incidence, survival, and mortality in North America. J Clin Oncol. 1992; 10: 323-9.

5. De Roos AJ, Olshan AF, Teschke K, Poole C, Savitz DA, Blatt J, et al. Parental occupational exposures to chemicals and incidence of neuroblastoma in offspring. Am J Epidemiol. 2001; 154: 106-14

6. De Roos AJ, Teschke K, Savitz DA, Poole C, Grufferman S, Pollock BH, et al. Parental occupational exposures to electromagnetic fields and radiation and the incidence of neuroblastoma in offspring. Epidemiology. 2001; 12: 508-17.

7. Capasso M, Diskin SJ. Genetics and genomics of neuroblastoma. Cancer Treat Res. 2010; 155: 65-84.

8. Chen Y, Takita J, Choi YL, Kato M, Ohira M, Sanada M, et al. Oncogenic mutations of ALK kinase in neuroblastoma. Nature. 2008; 455: 971-4.

9. George RE, Sanda T, Hanna M, Frohling S, Luther W, 2nd, Zhang J, et al. Activating mutations in ALK provide a therapeutic target in neuroblastoma. Nature. 2008; 455: 975-8.
10. Janoueix-Lerosey I, Lequin D, Brugieres L, Ribeiro A, de Pontual L, Combaret $\mathrm{V}$, et al. Somatic and germline activating mutations of the ALK kinase receptor in neuroblastoma. Nature. 2008; 455: 967-70.

11. Maris JM, Mosse YP, Bradfield JP, Hou C, Monni S, Scott RH, et al Chromosome 6p22 locus associated with clinically aggressive neuroblastoma. N Engl J Med. 2008; 358: 2585-93.

12. Han $\mathrm{W}$, Zhou $\mathrm{Y}$, Zhong $\mathrm{R}$, Wu C, Song $\mathrm{R}$, Liu $\mathrm{L}$, et al. Functional polymorphisms in FAS/FASL system increase the risk of neuroblastoma in Chinese population. PLoS One. 2013; 8: e71656.

13. Li J, Zou L, Zhou Y, Li L, Zhu Y, Yang Y, et al. A low-frequency variant in SMAD7 modulates TGF-beta signaling and confers risk for colorectal cancer in Chinese population. Mol Carcinog. 2017; 56: 1798-807.

14. Diskin SJ, Capasso M, Diamond M, Oldridge DA, Conkrite K, Bosse KR, et al. Rare variants in TP53 and susceptibility to neuroblastoma. J Natl Cancer Inst. 2014; 106: dju047.

15. Wang K, Diskin SJ, Zhang H, Attiyeh EF, Winter C, Hou C, et al. Integrative genomics identifies LMO1 as a neuroblastoma oncogene. Nature. 2011; 469: 216-20.

16. Matthews JM, Lester K, Joseph S, Curtis DJ. LIM-domain-only proteins in cancer. Nat Rev Cancer. 2013; 13: 111-22.

17. Oldridge DA, Wood AC, Weichert-Leahey N, Crimmins I, Sussman R, Winter $\mathrm{C}$, et al. Genetic predisposition to neuroblastoma mediated by a LMO1 super-enhancer polymorphism. Nature. 2015; 528: 418-21.

18. He J, Zhong $\mathrm{W}$, Zeng J, Zhu J, Zhang R, Wang F, et al. LMO1 gene polymorphisms contribute to decreased neuroblastoma susceptibility in a Southern Chinese population. Oncotarget. 2016; 7: 22770-8.

19. He J, Wang F, Zhu J, Zhang Z, Zou Y, Zhang R, et al. The TP53 gene rs 1042522 $\mathrm{C}>\mathrm{G}$ polymorphism and neuroblastoma risk in Chinese children. Aging (Albany NY). 2017; 9: 852-9.

20. He J, Zou Y, Wang T, Zhang R, Yang T, Zhu J, et al. Genetic Variations of GWAS-Identified Genes and Neuroblastoma Susceptibility: a Replication Study in Southern Chinese Children. Transl Oncol. 2017; 10: 936-41.

21. Zhang J, Lin H, Wang J, He J, Zhang D, Qin P, et al. LMO1 polymorphisms reduce neuroblastoma risk in Chinese children: a two-center case-control study. Oncotarget. 2017; 8: 65620-6.

22. Zhang J, Zhuo ZJ, Wang J, He J, Yang L, Zhang D, et al. CASC15 gene polymorphisms reduce neuroblastoma risk in Chinese children. Oncotarget. 2017; 8: 91343-9.

23. He J, Qiu LX, Wang MY, Hua RX, Zhang RX, Yu HP, et al. Polymorphisms in the XPG gene and risk of gastric cancer in Chinese populations. Hum Genet. 2012; 131: 1235-44.

24. Lou J, Gong J, Ke J, Tian J, Zhang Y, Li J, et al. A functional polymorphism located at transcription factor binding sites, rs6695837 near LAMC1 gene, confers risk of colorectal cancer in Chinese populations. Carcinogenesis. 2017; 38: 177-83.

25. He BS, Zhang LY, Pan YQ, Lin K, Zhang LL, Sun HL, et al. Association of the DISC1 and NRG1 genetic polymorphisms with schizophrenia in a Chinese population. Gene. 2016; 590: 293-7.

26. He BS, Sun HL, Xu T, Pan YQ, Lin K, Gao TY, et al. Association of Genetic Polymorphisms in the LncRNAs with Gastric Cancer Risk in a Chinese Population. J Cancer. 2017; 8: 531-6.

27. Li J, Wang Y, Zhu Y, Gong Y, Yang Y, Tian J, et al. Breast cancer risk-associated variants at 6q25.1 influence risk of hepatocellular carcinoma in a Chinese population. Carcinogenesis. 2017; 38: 447-54.

28. He J, Wang MY, Oiu LX, Zhu ML, Shi TY, Zhou XY, et al. Genetic variations of mTORC1 genes and risk of gastric cancer in an Eastern Chinese population. Mol Carcinog. 2013; 52 Suppl 1: E70-9.

29. He J, Wang F, Zhu J, Zhang R, Yang T, Zou Y, et al. Association of potentially functional variants in the XPG gene with neuroblastoma risk in a Chinese population. J Cell Mol Med. 2016; 20: 1481-90.

30. Wacholder S, Chanock S, Garcia-Closas M, El Ghormli L, Rothman N. Assessing the probability that a positive report is false: an approach for molecular epidemiology studies. J Natl Cancer Inst. 2004; 96: 434-42.

31. Rabbitts TH, Boehm T. LIM domains. Nature. 1990; 346: 418.

32. Boehm T, Foroni L, Kennedy M, Rabbitts TH. The rhombotin gene belongs to a class of transcriptional regulators with a potential novel protein dimerisation motif. Oncogene. 1990; 5: 1103-5.

33. Greenberg JM, Boehm T, Sofroniew MV, Keynes RJ, Barton SC, Norris ML, et al. Segmental and developmental regulation of a presumptive T-cell oncogene in the central nervous system. Nature. 1990; 344: 158-60.

34. Fagerberg L, Hallstrom BM, Oksvold P, Kampf C, Djureinovic D, Odeberg J, et al. Analysis of the human tissue-specific expression by genome-wide integration of transcriptomics and antibody-based proteomics. Mol Cell Proteomics. 2014; 13: 397-406.

35. Latorre V, Diskin SJ, Diamond MA, Zhang H, Hakonarson H, Maris JM, et al. Replication of neuroblastoma SNP association at the BARD1 locus in African-Americans. Cancer Epidemiol Biomarkers Prev. 2012; 21: 658-63.

36. Capasso M, Diskin SJ, Totaro F, Longo L, De Mariano M, Russo R, et al. Replication of GWAS-identified neuroblastoma risk loci strengthens the role of BARD1 and affirms the cumulative effect of genetic variations on disease susceptibility. Carcinogenesis. 2013; 34: 605-11.

37. Lu J, Chu P, Wang H, Jin Y, Han S, Han W, et al. Candidate Gene Association Analysis of Neuroblastoma in Chinese Children Strengthens the Role of LMO1. PLoS One. 2015; 10: e0127856. 